Revista Arbitrada Interdisciplinaria de Ciencias de la Salud. SALUD Y VIDA

Volumen 3. Número 6. Año 3. Julio - Diciembre 2019

Hecho el depósito de Ley: FA2016000010

ISSN: $2610-8038$

FUNDACIÓN KOINONIA (F.K)

Santa Ana de Coro, Venezuela.

Maholy Denisse Muñoz Macías; Nicolle Valeria Muñoz Macías; Juan Salvador Solorzano Intriago

DOI: http://dx.doi.org/10.35381/s.v.v3i6.337

\title{
Actualización sobre todos los avances en el campo de la cirugía
}

Update on all progress in the field of surgery

Maholy Denisse Muñoz Macías

Mademuma@hotmail.com

Universidad Laica Eloy Alfaro de Manabí

Ecuador

https://orcid.org/0000-0002-4757-594X

Nicolle Valeria Muñoz Macías

niky mm92@hotmail.com

Universidad Laica Eloy Alfaro de Manabí

Ecuador

https://orcid.org/0000-0001-6041-9995

Juan Salvador Solorzano Intriago

Juan issi91@hotmail.com

Universidad Laica Eloy Alfaro de Manabí

Ecuador

https://orcid.org/0000-0002-1175-0988

Recibido: 12 de mayo de 2019

Aprobado: 02 de junio de 2019

\section{RESUMEN}

Los nuevos centros de innovación quirúrgica están trabajando para definir el campo e identificar aspectos críticos de la promoción de la innovación quirúrgica. La responsabilidad como profesionales de la medicina es trabajar para comprender la innovación en cirugía, descubrir, traducir y comercializar avances para abordar problemas clínicos importantes y para apoyar el futuro de nuestra profesión de manera consistente y racional. El presente artículo de revisión contiene la síntesis de recientes avances en diferentes especialidades del campo de la cirugía que van desde la cirugía abdominal de pared y hernia, bariátrica, colorectal, debusto, gastrointestinal superior, endocrina, hepatobiliar y pancreática, de piel y tejido suave, de trauma y quemaduras, vascular y endovascular, el cuidado perioperativo, trasplantes así como otras especialidades quirúrgicas. 
Revista Arbitrada Interdisciplinaria de Ciencias de la Salud. SALUD Y VIDA

Volumen 3. Número 6. Año 3. Julio - Diciembre 2019

Hecho el depósito de Ley: FA2016000010

ISSN: $2610-8038$

FUNDACIÓN KOINONIA (F.K)

Santa Ana de Coro, Venezuela.

Maholy Denisse Muñoz Macías; Nicolle Valeria Muñoz Macías; Juan Salvador Solorzano Intriago

Descriptores: Cirugía; Avances; Ciencia; Operación; Desarrollo.

\begin{abstract}
The new surgical innovation centers are working to define the field and identify critical aspects of the promotion of surgical innovation. The responsibility as medical professionals is to work to understand innovation in surgery, discover, translate and market advances to address important clinical problems and to support the future of our profession in a consistent and rational manner. The present review article contains the synthesis of recent advances in different specialties in the field of surgery ranging from abdominal wall surgery and hernia, bariatric, colorectal, debut, upper gastrointestinal, endocrine, hepatobiliary and pancreatic, skin and soft tissue, trauma and burns, vascular and endovascular, perioperative care, transplants as well as other surgical specialties.
\end{abstract}

Descriptors: Surgery; Advances; Science; Operation; Development.

\title{
RECIENTES AVANCES EN DIFERENTES ESPECIALIDADES QUIRÚRGICAS CIRUGÍA ABDOMINAL DE PARED Y HERNIA
}

Reparación laparoscópica versus abierta de hernias inguinales primarias (junio 2019)

La reparación de la hernia inguinal se puede realizar abierta o laparoscópicamente. En un ensayo aleatorizado de más de 400 adultos con hernias inguinales primarias, la reparación laparoscópica extraperitoneal total (TEP) tuvo una menor morbilidad y una recuperación más rápida después de la operación en comparación con la reparación abierta de Lichtenstein; sin embargo, la satisfacción del paciente, el dolor en la ingle y las tasas de recurrencia fueron similares en uno y tres años (Gutlic et al., 2019). Para hernias inguinales primarias, unilaterales, es aceptable la reparación abierta o laparoscópica; Los cirujanos deben elegir la técnica para la que tienen más experiencia. 
Revista Arbitrada Interdisciplinaria de Ciencias de la Salud. SALUD Y VIDA

Volumen 3. Número 6. Año 3. Julio - Diciembre 2019

Hecho el depósito de Ley: FA2016000010

ISSN: $2610-8038$

FUNDACIÓN KOINONIA (F.K)

Santa Ana de Coro, Venezuela.

Maholy Denisse Muñoz Macías; Nicolle Valeria Muñoz Macías; Juan Salvador Solorzano Intriago

Problemas con la malla intraperitoneal para la prevención de la hernia incisional (febrero de 2019)

Las hernias incisionales ocurren en aproximadamente 10 a 15 por ciento de los pacientes después de una incisión abdominal; La malla colocada en el momento del cierre abdominal puede reducir esta tasa. En un ensayo aleatorizado, 150 pacientes con alto riesgo de desarrollar hernias incisionales se sometieron a un cierre abdominal con o sin implante de malla intraperitoneal (Kohler et al., 2019). Aunque la malla redujo la incidencia de hernia a los tres años, también contribuyó a aumentar el dolor abdominal en el postoperatorio temprano y retrasó la curación después de una infección del sitio quirúrgico. Dados los posibles efectos nocivos, sugerimos no colocar la malla rutinariamente en el cierre abdominal.

\section{CIRUGÍA BARIÁTRICA}

\section{Resultados de la cirugía bariátrica en adolescentes (junio de 2019)}

El efecto de la edad del paciente en el momento de la cirugía bariátrica sobre el impacto beneficioso del procedimiento no está claro. En un análisis de los datos de dos estudios de cohorte observacionales prospectivos realizados en más de 160 adolescentes y casi 400 adultos que tenían bypass gástrico Roux-en-Y (RYGB) para obesidad severa, a los cinco años, los adolescentes tenían más probabilidades que los adultos de experimentar remisión. de diabetes tipo 2 (86 versus 53 por ciento) e hipertensión (68 versus 41 por ciento); el porcentaje de pérdida de peso fue similar (-26 versus -29 por ciento) (Inge et al., 2019) Estos hallazgos aumentan la posibilidad de que una intervención temprana con RYGB durante la adolescencia pueda tener mayores beneficios metabólicos a largo plazo que la cirugía durante la edad adulta, aunque se necesitan más datos para determinar si se mantendrá la remisión de diabetes o hipertensión. Además, estos hallazgos pueden no reflejar los resultados de la gastrectomía en manga, que ahora es la forma más común de cirugía bariátrica en los Estados Unidos. 


\section{Eficacia y complicaciones de un bypass gástrico de anastomosis versus bypass} gástrico Roux-en-Y estándar (abril de 2019)

Una anastomosis bypass gástrico ( $\mathrm{AOBG}$ ) es un procedimiento quirúrgico bariátrico que está ganando popularidad internacionalmente, pero no en los Estados Unidos. En un ensayo que incluyó a más de 250 adultos obesos con al menos una comorbilidad, AOBG resultó en una pérdida de peso similar al bypass gástrico Roux-en-Y (RYGB) estándar y una mayor mejora en la hemoglobina glucosilada (A1C) en el subgrupo con diabetes (Robert et al., 2019). Sin embargo, AOBG también resultó en más complicaciones relacionadas con la malabsorción (por ejemplo, deficiencia nutricional, diarrea, esteatorrea), posiblemente debido al uso de una extremidad biliopancreática más larga $(200 \mathrm{~cm})$. Hasta que se resuelva el problema de la malabsorción excesiva, estamos de acuerdo con la Sociedad Estadounidense de Cirugía Metabólica y Bariátrica de que la $A O B G$ debe seguir siendo un procedimiento de investigación.

\section{CIRUGÍA COLORECTAL}

Cirugía colorrectal mínimamente invasiva con recuperación mejorada después del protocolo (ERAS) y telemedicina (agosto de 2019)

Aunque los protocolos de recuperación mejorada después de la cirugía (ERAS) reducen la duración de la estadía (LOS) después de la cirugía colorrectal abierta, se debate su efecto sobre la cirugía mínimamente invasiva. En un ensayo que asignó al azar a 63 pacientes sometidos a cirugía colorrectal mínimamente invasiva a un protocolo ERAS estandarizado con un programa de telemedicina estructurado o atención postoperatoria estándar, la mediana de LOS total de 30 días (ingreso índice más cualquier reingreso) en los dos grupos fue de aproximadamente 28 y 52 horas, respectivamente (Bednarski et al., 2019). Cuatro pacientes en el grupo ERAS y uno en el grupo control requirieron una visita a la sala de emergencias o reingreso, pero ninguno por razones relacionadas con el alta acelerada. Si se valida, una estrategia 
Revista Arbitrada Interdisciplinaria de Ciencias de la Salud. SALUD Y VIDA

Volumen 3. Número 6. Año 3. Julio - Diciembre 2019

Hecho el depósito de Ley: FA2016000010

ISSN: $2610-8038$

FUNDACIÓN KOINONIA (F.K)

Santa Ana de Coro, Venezuela.

Maholy Denisse Muñoz Macías; Nicolle Valeria Muñoz Macías; Juan Salvador Solorzano Intriago

que combine cirugía mínimamente invasiva y un protocolo ERAS con telemedicina puede permitir la cirugía colorrectal ambulatoria (es decir, de corta duración o "observación de 23 horas").

\section{Colonoscopia después de diverticulitis aguda (julio de 2019)}

En un metaanálisis de 17 estudios observacionales de colonoscopia después de diverticulitis aguda, se detectó cáncer colorrectal en 2.1 por ciento (IC 95\% 1.5-3.1) de pacientes (Rottier et al., 2019). Este riesgo es mayor que la prevalencia de cáncer colorrectal de 0.4 a 1.0 por ciento en la población general que se somete a una colonoscopia de detección. Estos datos brindan apoyo adicional para nuestra recomendación de colonoscopia en todos los pacientes después de un episodio de diverticulitis aguda, a menos que el procedimiento se haya realizado en el año anterior.

\section{Ligadura alta versus baja de la arteria mesentérica inferior durante la cirugía de cáncer rectal (junio de 2019)}

En la cirugía de cáncer rectal, la ligadura alta versus baja de la arteria mesentérica inferior (IMA) es controvertida. En el ensayo HIGHLOW de más de 200 pacientes sometidos a resección anterior baja laparoscópica, en comparación con ligar el IMA en su origen, la ligadura debajo del despegue de la arteria cólica izquierda resultó en menos síntomas urinarios obstructivos y una mejor preservación de la continencia urinaria, la función sexual y la calidad. de vida a los nueve meses después de la operación (Mari et al., 2019). Las tasas de complicaciones (p. Ej., Fuga anastomótica) y los resultados oncológicos a corto plazo fueron similares para ambos enfoques. En pacientes con cáncer rectal pero sin enfermedades nodales en la raíz del IMA, preferimos ligar el IMA debajo del despegue de la arteria cólica izquierda. 
Revista Arbitrada Interdisciplinaria de Ciencias de la Salud. SALUD Y VIDA

Volumen 3. Número 6. Año 3. Julio - Diciembre 2019

Hecho el depósito de Ley: FA2016000010

ISSN: $2610-8038$

FUNDACIÓN KOINONIA (F.K)

Santa Ana de Coro, Venezuela.

Maholy Denisse Muñoz Macías; Nicolle Valeria Muñoz Macías; Juan Salvador Solorzano Intriago

Anastomosis grapada versus cosida a mano para hemicolectomía derecha (mayo de 2019)

La anastomosis ileocólica después de la hemicolectomía derecha se puede realizar con engrapadoras o suturas. Aunque un metanálisis de ensayos aleatorios encontró una tasa menor de fugas con anastomosis engrapadas en comparación con las cosidas a mano, varios estudios observacionales grandes posteriores informaron hallazgos opuestos. En un reciente estudio observacional de más de 1400 pacientes sometidos a resección de colon por cáncer del lado derecho, las anastomosis con grapas se asociaron con una tasa de fuga más alta que las anastomosis cosidas a mano (5.4 versus 2.4 por ciento) (Nordholm, Schnack y Krarup, 2019). Hasta que los datos concluyentes demuestren la superioridad de una técnica, recomendamos que los cirujanos se vuelvan fáciles con ambas técnicas y adapten su uso a cada situación individual del paciente.

\section{Antibióticos de rutina después del drenaje del absceso anorrectal (abril de 2019)}

Después de un procedimiento de drenaje para un absceso anorrectal, las complicaciones pueden incluir el desarrollo de una fístula o la formación de abscesos recurrentes. El papel de los antibióticos para prevenir complicaciones ha sido incierto, y las principales directrices de la sociedad recomiendan el tratamiento solo para pacientes de alto riesgo. Un metanálisis de 2019 de dos ensayos aleatorios y un estudio retrospectivo encontró que la terapia antibiótica empírica de rutina se asoció con una tasa más baja de formación de fístulas (16 versus 24 por ciento), aunque la calidad de la evidencia no fue alta (riesgo de sesgo y alta grado de heterogeneidad) (Mocanu et al. 2019) No hubo ningún efecto sobre la tasa de recurrencia de abscesos, pero, dada la significativa morbilidad asociada con la fistulización, ahora sugerimos antibióticos empíricos de rutina para todos los pacientes que siguen un drenaje de absceso anorrectal. En general, prescribimos un curso de cuatro a cinco días de amoxicilina-clavulanato o ciprofloxacina / metronidazol. 
Revista Arbitrada Interdisciplinaria de Ciencias de la Salud. SALUD Y VIDA

Volumen 3. Número 6. Año 3. Julio - Diciembre 2019

Hecho el depósito de Ley: FA2016000010

ISSN: $2610-8038$

FUNDACIÓN KOINONIA (F.K)

Santa Ana de Coro, Venezuela.

Maholy Denisse Muñoz Macías; Nicolle Valeria Muñoz Macías; Juan Salvador Solorzano Intriago

Colocación de malla no es efectiva en la prevención de la hernia paraestomal (abril de 2019)

Las hernias parastomales ocurren en hasta el 50 por ciento de los pacientes con colostomía o ileostomía. Con base en los resultados de un ensayo anterior (PREVENIR) que informó un beneficio para la colocación de malla profiláctica en el momento de la construcción del estoma, previamente habíamos recomendado el uso rutinario de malla profiláctica. Un ensayo más grande y más reciente (STOMAMESH) ahora informa que la malla profiláctica no redujo la incidencia de hernia parastomal al año (Odensten et al. 2019). PREVENIR y STOMAMESH tuvieron diseños similares pero, a diferencia del ensayo anterior, la evaluación clínica en STOMAMESH incluyó el cegamiento a la intervención y el seguimiento de la tomografía computarizada de rutina para evaluar la hernia. Dado el potencial de complicaciones raras relacionadas con la malla, y que el único beneficio de la malla profiláctica para prevenir la hernia paraestomal está en duda, ahora sugerimosno coloca rutinariamente la malla en el momento de la construcción permanente del estoma.

Beneficio de los estomas de derivación temporales en la cirugía de cáncer rectal y el momento óptimo de cierre (abril de 2019)

Los estomas de derivación temporales se realizan comúnmente para proteger las anastomosis colorrectales después de la cirugía de cáncer rectal. Dos estudios abordaron su beneficio y el momento óptimo de cierre:

- En un estudio de base de datos administrativa de más de 8600 proctectomías con preservación del esfínter, la desviación temporal se asoció con mayores tasas de reintervenciones y reingresos y un mayor costo, pero no menos fugas (Chapman et al., 2019). Las limitaciones de diseño en el estudio, incluida la falta de estratificación por distancia entre la anastomosis y el borde anal o la gravedad de la fuga, pueden explicar en parte la aparente falta de beneficio de la derivación temporal. 
Revista Arbitrada Interdisciplinaria de Ciencias de la Salud. SALUD Y VIDA

Volumen 3. Número 6. Año 3. Julio - Diciembre 2019

Hecho el depósito de Ley: FA2016000010

ISSN: $2610-8038$

FUNDACIÓN KOINONIA (F.K)

Santa Ana de Coro, Venezuela.

Maholy Denisse Muñoz Macías; Nicolle Valeria Muñoz Macías; Juan Salvador Solorzano Intriago

-En un ensayo que comparó el cierre temprano (8 a 13 días) con el cierre tardío (después de 12 semanas) de un estoma de derivación temporal en 112 pacientes que se sometieron a cirugía de cáncer rectal, el cierre temprano redujo la suciedad fecal y la cantidad de estomas permanentes (Keane et al., 2019). Además, hubo una tendencia hacia una menor incidencia de síndrome de resección anterior baja importante (SRAB) con cierre temprano.

Reservamos el estoma de derivación temporal para anastomosis bajas $(<5 \mathrm{~cm}$ desde el borde anal) y pacientes que han sido tratados con radiación pélvica preoperatoria o terapia inmunosupresora. Para los pacientes que no requieren quimioterapia adyuvante, los estomas pueden cerrarse a las tres semanas, pero el tiempo exacto queda a discreción del cirujano. Para pacientes con un tumor localmente avanzado o ganglios linfáticos regionales positivos, sugerimos cerrar el estoma después de que se haya completado la quimioterapia adyuvante.

\section{Seguimiento de dos años de ensayos aleatorios que comparan la cirugía laparoscópica con cirugía abierta de cáncer rectal (abril de 2019)}

En 2015, dos ensayos aleatorios (ACOSOG y AlaCaRT) en pacientes con cáncer rectal encontraron que la cirugía laparoscópica resultó en tasas más altas de escisión mesorrectal incompleta que la cirugía abierta. Ahora, los datos de seguimiento a los dos años muestran una tendencia no estadísticamente significativa hacia una mejor supervivencia y menos recurrencias locales después de la cirugía abierta (Fleshman et al., 2019; Stevenson et al., 2019). Se necesitan datos a largo plazo para determinar si la cirugía abierta resulta en una verdadera ventaja de supervivencia. Hasta que estos datos estén disponibles, la cirugía laparoscópica para el cáncer de recto sigue siendo una opción, pero solo debe ser realizada por cirujanos con experiencia en técnicas de escisión mesorrectal total mínimamente invasivas y en pacientes sin un margen circunferencial amenazado o de alto riesgo. 
Revista Arbitrada Interdisciplinaria de Ciencias de la Salud. SALUD Y VIDA

Volumen 3. Número 6. Año 3. Julio - Diciembre 2019

Hecho el depósito de Ley: FA2016000010

ISSN: $2610-8038$

FUNDACIÓN KOINONIA (F.K)

Santa Ana de Coro, Venezuela.

Maholy Denisse Muñoz Macías; Nicolle Valeria Muñoz Macías; Juan Salvador Solorzano Intriago

Apendicectomía de intervalo después del tratamiento no quirúrgico de la apendicitis perforada (marzo de 2019)

Si bien la mayoría de los pacientes estables con apendicitis perforada se pueden manejar de forma no operativa en el momento de la presentación inicial con antibióticos intravenosos y drenaje percutáneo de un absceso (si está presente), es controvertido si se debe realizar una apendicectomía de intervalo. En un ensayo aleatorizado en el que participaron 60 pacientes adultos con absceso periappendicular que respondieron al tratamiento inicial no quirúrgico, los pacientes fueron asignados a una apendicectomía a intervalos o un seguimiento por resonancia magnética (Mällinen et al., 2019). El ensayo se terminó antes de tiempo debido a una alta incidencia de neoplasias apendiculares, y a todos los pacientes se les ofreció apendicectomía. Se detectó neoplasia apendicular en el 20 por ciento de todos los pacientes y el 29 por ciento de los pacientes mayores de 40 años. Esta prevalencia, en línea con las estimaciones de series anteriores de muestras de apendicectomía de intervalo (10 a 29 por ciento), es sustancialmente mayor que la de los pacientes con apendicitis no complicada (1 por ciento). Ahora sugerimos la apendicectomía de intervalo para todos los pacientes con apendicitis perforada manejados de forma no operativa.

\section{Procedimiento de Hartmann versus anastomosis primaria con derivación proximal para diverticulitis perforada (marzo de 2019)}

La diverticulitis sigmoidea perforada aguda se puede tratar con el procedimiento de Hartmann o con anastomosis primaria con derivación proximal (APDP). Aunque los ensayos pequeños han demostrado resultados iguales o superiores con APDP, un estudio de base de datos que comparó los dos enfoques quirúrgicos en más de 10,000 pacientes encontró tasas de mortalidad y morbilidad mucho más altas para APDP cuando fueron realizadas por cirujanos no colorrectales (Goldstone et al, 2019). Para los cirujanos generales que realizan la mayoría de las cirugías para la diverticulitis perforada, sugerimos el procedimiento de Hartmann sobre la APDP. 
Revista Arbitrada Interdisciplinaria de Ciencias de la Salud. SALUD Y VIDA

Volumen 3. Número 6. Año 3. Julio - Diciembre 2019

Hecho el depósito de Ley: FA2016000010

ISSN: $2610-8038$

FUNDACIÓN KOINONIA (F.K)

Santa Ana de Coro, Venezuela.

Maholy Denisse Muñoz Macías; Nicolle Valeria Muñoz Macías; Juan Salvador Solorzano Intriago

\section{CIRUGÍA DE BUSTO}

Retiro del mercado de ciertos implantes mamarios con textura y expansores de tejidos (julio de 2019)

Los implantes mamarios con superficie texturizada se han asociado con un mayor riesgo de linfoma anaplásico de células grandes. La evidencia de que un producto específico del fabricante parecía estar directamente relacionado con el daño al paciente llevó a la Administración de Drogas y Alimentos de los Estados Unidos (ADA) a solicitar un retiro voluntario de ciertos implantes con textura (Administración de Drogas y Alimentos de los Estados Unidos, ADA 2019b). El retiro del mercado incluye implantes permanentes y expansores de tejido con la superficie BIOCELL, pero no incluye dispositivos de superficie lisa que usan solución salina o gel de silicona como material de relleno. La $A D A$ no recomienda la extracción de implantes para mujeres asintomáticas que tienen un implante mamario texturizado.

\section{Óxido de hierro superparamagnético en el tratamiento de los ganglios axilares en el carcinoma ductal in situ de alto riesgo (abril de 2019)}

En pacientes con carcinoma ductal in situ (CDIS) con alta sospecha clínica de cáncer invasivo sincrónico, la biopsia de ganglio linfático centinela (BGLC) se puede realizar de forma rutinaria en la cirugía primaria de mama o selectivamente como una segunda cirugía en pacientes con enfermedad invasiva confirmada. En un estudio de pacientes con CDIS de alto riesgo en los que se inyectó óxido de hierro superparamagnético (OHSM) como marcador en el momento de la mastectomía o la cirugía de conservación del seno, el 78 por ciento de los pacientes no tenían enfermedad invasiva y, por lo tanto, pudieron evitar una segunda cirugía para BGLC (Karakatsanis et al., 2019) En el resto que requirió una nueva operación para BGLC, la señal OHSM persistió hasta un mes después de la inyección y (en combinación con el tinte azul) superó al isótopo más el tinte azul en la detección de ganglios centinela. Si la seguridad y la eficacia de 
Revista Arbitrada Interdisciplinaria de Ciencias de la Salud. SALUD Y VIDA

Volumen 3. Número 6. Año 3. Julio - Diciembre 2019

Hecho el depósito de Ley: FA2016000010

ISSN: $2610-8038$

FUNDACIÓN KOINONIA (F.K).

Santa Ana de Coro, Venezuela.

Maholy Denisse Muñoz Macías; Nicolle Valeria Muñoz Macías; Juan Salvador Solorzano Intriago

OHSM se validan en estudios prospectivos, este enfoque puede mejorar el tratamiento axilar en pacientes con DCIS de alto riesgo.

\section{CIRUGÍA ENDOCRINA}

Tiroidectomía en hipotiroidismo autoinmune con síntomas persistentes (abril de 2019)

Los síntomas persistentes en pacientes tratados con levotiroxina con hipotiroidismo mediado por autoinmunidad pueden deberse a los anticuerpos de la peroxidasa tiroidea (TPO), o a los moduladores inmunológicos asociados, que causan inflamación en otros tejidos además de la tiroides. En un ensayo de 18 meses que evaluó a 150 pacientes con tiroiditis de Hashimoto que presentaban síntomas persistentes a pesar del reemplazo óptimo de la hormona tiroidea y la hormona estimulante de la tiroides (TSH) y tiroxina libre (T4), tiroidectomía total (para eliminar el estímulo antigénico y, por lo tanto, reducir el autoanticuerpo tiroideo) niveles) disminuyeron los niveles de anticuerpos TPO y mejoraron el resultado primario (puntaje de salud general SF-36) en comparación con los pacientes que no se sometieron a cirugía (Guldvog et al., 2019) La ausencia de un grupo quirúrgico simulado limita la validez de este estudio, y es probable que este hallazgo provocador siga siendo controvertido. La tiroidectomía tiene complicaciones quirúrgicas (infección, hipocalcemia, parálisis recurrente del nervio laríngeo) y no es un tratamiento recomendado para la tiroiditis mediada por autoinmunidad.

\section{CIRUGÍA HEPATOBILIAR Y PANCREÁTICA}

\section{Dolor abdominal después de la colecistectomía (julio de 2019)}

Una parte significativa de los pacientes que se someten a colecistectomía se quejan de dolor abdominal después. En un ensayo de más de 1000 pacientes con dolor abdominal y cálculos biliares o lodo probados por ultrasonido, tanto la atención habitual (colecistectomía a discreción del cirujano) como un enfoque restrictivo (selección de 
Revista Arbitrada Interdisciplinaria de Ciencias de la Salud. SALUD Y VIDA

Volumen 3. Número 6. Año 3. Julio - Diciembre 2019

Hecho el depósito de Ley: FA2016000010

ISSN: $2610-8038$

FUNDACIÓN KOINONIA (F.K)

Santa Ana de Coro, Venezuela.

Maholy Denisse Muñoz Macías; Nicolle Valeria Muñoz Macías; Juan Salvador Solorzano Intriago

pacientes para cirugía basada en la presencia de criterios consistentes con cólico biliar) dieron como resultado tasas similares de dolor abdominal a los 12 meses (40 y 44 por ciento, respectivamente, en general; 37 y 36 por ciento, respectivamente, de los que se sometieron a colecistectomía) (van Dijk et al., 2019) Como los cálculos biliares y el dolor abdominal son afecciones comunes, se necesitan pruebas de diagnóstico que permitan una identificación más precisa de aquellos pacientes con cálculos biliares que se beneficiarán de la colecistectomía. Hasta que tales pruebas estén disponibles, los cirujanos deben advertir a los pacientes que la colecistectomía puede no aliviar su dolor.

Resultados a 10 años de la pancreatectomía total con autotrasplante de células de los islotes para la pancreatitis crónica (mayo de 2019)

En un estudio observacional en un solo centro de más de 200 pacientes con pancreatitis crónica que se sometieron a pancreatectomía total con autotrasplante de islotes (PTATI), el 82 por ciento informó un alivio sostenido del dolor, el 20 por ciento era independiente de la insulina y el 32 por ciento tenía al menos una función de injerto parcial a los 10 años (Bellin et al., 2019). El trasplante> 4000 equivalentes de islotes / kilogramo de peso corporal fue el mejor predictor de la función del injerto. Estos hallazgos apoyan PTATI como el tratamiento preferido para la pancreatitis crónica de conducto pequeño con afectación parenquimatosa difusa. Sin embargo, debido a la insuficiente reserva de células de los islotes, pocos pacientes adultos son candidatos para el procedimiento.

\section{Pancreatectomía distal con una grapadora reforzada versus una engrapadora desnuda (abril de 2019)}

Durante la pancreatectomía distal, el páncreas se puede cortar con una grapadora. En un ensayo anterior, el refuerzo de malla de la engrapadora redujo la incidencia de fugas pancreáticas clínicamente importantes en comparación con la ausencia de malla. En 
Revista Arbitrada Interdisciplinaria de Ciencias de la Salud. SALUD Y VIDA

Volumen 3. Número 6. Año 3. Julio - Diciembre 2019

Hecho el depósito de Ley: FA2016000010

ISSN: $2610-8038$

FUNDACIÓN KOINONIA (F.K)

Santa Ana de Coro, Venezuela.

Maholy Denisse Muñoz Macías; Nicolle Valeria Muñoz Macías; Juan Salvador Solorzano Intriago

contraste, un ensayo reciente (HiSCO-07) encontró que el refuerzo de malla, en comparación con una engrapadora desnuda, no redujo la incidencia de fugas pancreáticas en la población general, pero lo hizo en pacientes con un páncreas (Kondo et al., 2019). Dados los datos en conflicto, el uso de refuerzo de malla debe dejarse a discreción del cirujano. Utilizamos una grapadora de triple altura que rara vez requiere refuerzo.

Resultados adversos de la pancreaticoduodenectomía laparoscópica versus abierta (marzo de 2019)

Aunque los estudios observacionales y los ensayos pequeños han establecido la viabilidad de la pancreaticoduodenectomía laparoscópica, en un ensayo reciente de 105 pacientes, este enfoque dio lugar a aumentos no estadísticamente significativos en las complicaciones postoperatorias mayores y las tasas de mortalidad relacionadas con complicaciones en comparación con la cirugía abierta, sin mejoría en la recuperación funcional (Comité de redacción para el grupo colaborativo PROBESE de la red de ventilación PROtective (PROVEnet) para la red de ensayos clínicos de la Sociedad Europea de Anestesiología et al, 20019). Estas tendencias resultaron en la terminación anticipada de la prueba. Teniendo en cuenta los datos pertinentes, sugerimos ofrecer una pancreaticoduodenectomía mínimamente invasiva con precaución, y solo en centros de gran volumen donde las complicaciones se puedan tratar fácilmente.

\section{CUIDADO PERIOPERATIVO}

\section{Ventilación intraoperatoria de protección pulmonar para pacientes obesos (agosto de 2019)}

La ventilación protectora pulmonar con volúmenes corrientes bajos, presión espiratoria final positiva alta (PEEP) y maniobras de reclutamiento mejora la oxigenación y la mecánica pulmonar en pacientes obesos, pero se desconoce el nivel óptimo de PEEP y los efectos de la ventilación protectora pulmonar en las complicaciones pulmonares 
postoperatorias. PROBESE fue un gran ensayo multicéntrico internacional con más de 2000 pacientes obesos con riesgo moderado o alto de complicaciones pulmonares postoperatorias que se sometieron a cirugía que duró> 2 horas (Comité de redacción para el grupo colaborativo PROBESE de la red de ventilación PROtective (PROVEnet) para la red de ensayos clínicos de la Sociedad Europea de Anestesiología, 2019). Los pacientes fueron asignados aleatoriamente para recibir PEEP a $4 \mathrm{~cm} \mathrm{H} 2 \mathrm{O}$ sin maniobras de reclutamiento, $012 \mathrm{~cm} \mathrm{H} 20$ PEEP con maniobras de reclutamiento; todos los pacientes recibieron $7 \mathrm{ml} / \mathrm{kg}$ de volumen corriente. No hubo diferencias en las complicaciones pulmonares postoperatorias entre los grupos. Si bien el ensayo PROBESE no respalda una estrategia de ventilación uniforme para pacientes obesos, sugerimos el uso de bajos volúmenes de marea, PEEP individualizada para optimizar la meseta y la presión de conducción, y el uso juicioso de las maniobras de reclutamiento.

\section{Un nuevo modelo de riesgo cardiovascular preoperatorio derivado y validado (junio de 2019)}

Aunque todos los pacientes sometidos a cirugía no cardíaca deben someterse a una evaluación preoperatoria del riesgo cardiovascular, no se ha identificado una herramienta óptima. El estudio de evaluación cardiovascular preoperatoria de la Universidad de Beirut de los Estados Unidos derivó y validó prospectivamente un nuevo índice de riesgo cardiovascular preoperatorio, denominado IRCV (Dakik et al., 2019). Se identificaron seis factores de riesgo en el análisis multivariado (edad $\geq 75$ años, antecedentes de enfermedad cardíaca, síntomas de angina o disnea, hemoglobina $<12 \mathrm{mg} / \mathrm{dL}$, cirugía vascular y cirugía de emergencia) y juntos podrían usarse para predecir con éxito el riesgo cardiovascular. La herramienta se comparó bien con el modelo IRCV de uso común. Se necesitan estudios de validación adicionales antes de recomendar este nuevo modelo de riesgo. 
Revista Arbitrada Interdisciplinaria de Ciencias de la Salud. SALUD Y VIDA

Volumen 3. Número 6. Año 3. Julio - Diciembre 2019

Hecho el depósito de Ley: FA2016000010

ISSN: $2610-8038$

FUNDACIÓN KOINONIA (F.K)

Santa Ana de Coro, Venezuela.

Maholy Denisse Muñoz Macías; Nicolle Valeria Muñoz Macías; Juan Salvador Solorzano Intriago

Riesgo cardiovascular perioperatorio en pacientes con apnea obstructiva del sueño (mayo de 2019)

Varios estudios prospectivos pequeños en pacientes con apnea obstructiva del sueño (AOS) han demostrado un mayor riesgo cardiovascular (CV) en el período perioperatorio. Ahora, en el estudio prospectivo más grande hasta la fecha, el aumento del riesgo CV se confirmó en 1200 pacientes sometidos a cirugía mayor no cardíaca (Chan et al., 2019). La AOS y, en particular, la AOS grave, fue un factor de riesgo independiente para eventos CV que incluyeron lesiones miocárdicas postoperatorias, muerte cardíaca, insuficiencia cardíaca congestiva, tromboembolismo, fibrilación auricular y accidente cerebrovascular. Se desconoce si las intervenciones pueden reducir este riesgo.

\section{Reprogramación de DAl para procedimientos quirúrgicos seleccionados (abril de 2019)}

El riesgo de interferencia electromagnética intraoperatoria (IEMI) con la función de un desfibrilador cardioversor implantable $(D C l)$ es mayor si se usa una unidad de electrocirugía monopolar (ESU) cerca del generador de pulso o los cables del DCI. En un estudio de cohorte prospectivo, el uso de ESU monopolar causó EMI clínicamente significativo (definido como interferencia que habría resultado en la administración de una terapia antitaquicardia inapropiada [estimulación o shock antitaquicardia] si el DCl no hubiera sido reprogramado) en el 20 por ciento de los pacientes sometidos a cirugía no cardíaca por encima del ombligo y en el 29 por ciento de los que se someten a cirugía cardíaca; La IEMI no ocurrió si la cirugía estaba debajo del ombligo (Schulman et al., 2019) Estos datos respaldan las recomendaciones actuales de manejo para la reprogramación de $\mathrm{DCl}$ para suspender la terapia antitaquicardia poco antes de cualquier procedimiento quirúrgico por encima del ombligo si se planea el uso de ESU. 
Revista Arbitrada Interdisciplinaria de Ciencias de la Salud. SALUD Y VIDA

Volumen 3. Número 6. Año 3. Julio - Diciembre 2019

Hecho el depósito de Ley: FA2016000010

ISSN: $2610-8038$

FUNDACIÓN KOINONIA (F.K)

Santa Ana de Coro, Venezuela.

Maholy Denisse Muñoz Macías; Nicolle Valeria Muñoz Macías; Juan Salvador Solorzano Intriago

Riesgo de cirugía no cardíaca en pacientes con insuficiencia cardíaca (marzo de 2019)

La insuficiencia cardíaca (IC) es un importante factor de riesgo de eventos adversos, incluida la muerte, después de la cirugía. Sin embargo, no se ha establecido el riesgo en pacientes con solo síntomas previos de insuficiencia cardíaca. Un estudio retrospectivo de más de 600,000 adultos (casi 50,000 con insuficiencia cardíaca) sometidos a cirugía electiva no cardíaca encontró que las tasas de mortalidad postoperatoria a los 90 días fueron más altas en pacientes con síntomas actuales de insuficiencia cardíaca en comparación con aquellos con síntomas anteriores de insuficiencia cardíaca (Lerman et al., 2019). Sin embargo, en comparación con pacientes sin antecedentes de insuficiencia cardíaca, el riesgo de mortalidad postoperatoria fue mayor en pacientes con síntomas previos de insuficiencia cardíaca, incluidos aquellos con función sistólica ventricular izquierda preservada.

\section{Ningún beneficio del baño universal de clorhexidina en pacientes hospitalizados en la UCl (marzo de 2019)}

El baño de clorhexidina de rutina reduce las infecciones adquiridas en el hospital en pacientes en unidades de cuidados intensivos, pero no hay datos suficientes para respaldar esta práctica en las unidades médicas y quirúrgicas generales. En un ensayo aleatorizado por conglomerados que incluyó a más de 330,000 pacientes en 53 hospitales de los Estados Unidos, el baño universal de clorhexidina (con mupirocina dirigida para el transporte de Staphylococcus aureus resistente a la meticilina[MRSA]) no redujo las tasas de infección adquirida en el hospital en comparación con la atención estándar durante un período de intervención de 21 meses (Huang et al., 2019). Sin embargo, en el análisis post-hoc de pacientes con dispositivos médicos, se asoció con reducciones en las tasas de infección; por lo tanto, se justifica un estudio prospectivo adicional del baño de clorhexidina para este subconjunto de pacientes. 
Revista Arbitrada Interdisciplinaria de Ciencias de la Salud. SALUD Y VIDA

Volumen 3. Número 6. Año 3. Julio - Diciembre 2019

Hecho el depósito de Ley: FA2016000010

ISSN: $2610-8038$

FUNDACIÓN KOINONIA (F.K)

Santa Ana de Coro, Venezuela.

Maholy Denisse Muñoz Macías; Nicolle Valeria Muñoz Macías; Juan Salvador Solorzano Intriago

\section{CIRUGÍA DE PIEL Y TEJIDO SUAVE}

Resultados a largo plazo de márgenes de resección de 2 versus $4 \mathrm{~cm}$ para melanoma cutáneo (julio de 2019)

Para el melanoma cutáneo localizado más grueso que $2 \mathrm{~mm}$ del tronco o las extremidades, el margen mínimo de resección seguro sugerido es de $2 \mathrm{~cm}$ en las guías de todo el mundo. En un ensayo aleatorizado que comparó un margen de resección de 2 versus $4 \mathrm{~cm}$ en más de 900 pacientes, la supervivencia general y específica del melanoma fue similar en ambos grupos con una mediana de seguimiento de 19,6 años (Utjés et al., 2019). Estos datos a largo plazo respaldan las recomendaciones de margen actuales para melanomas primarios $>2 \mathrm{~mm}$ de espesor.

\section{Inhibidores de SGLT2 y fascitis necrotizante del perineo (mayo de 2019)}

En pacientes con diabetes tipo 2, los inhibidores del co-transportador de sodio-glucosa 2 (SGLT2) reducen la glucosa en sangre al aumentar la excreción urinaria de glucosa. Los efectos adversos incluyen una mayor incidencia de infecciones genitales y del tracto urinario. Hay un número creciente de informes de fascitis necrosante del perineo (gangrena de Fournier) en pacientes que toman inhibidores de SGLT2 (55 casos desde que los inhibidores de SGLT2 estuvieron disponibles hace seis años en comparación con 19 casos en 35 años entre pacientes tratados con otras clases de agentes para la diabetes) (Bersoff et al., 2019) Los casos ocurrieron entre 5 días y 49 meses después del inicio de los inhibidores de SGLT2. Los médicos deben ser conscientes de esta complicación potencialmente mortal, ya que la identificación y el tratamiento tempranos pueden reducir la morbilidad y la mortalidad. 
Revista Arbitrada Interdisciplinaria de Ciencias de la Salud. SALUD Y VIDA

Volumen 3. Número 6. Año 3. Julio - Diciembre 2019

Hecho el depósito de Ley: FA2016000010

ISSN: $2610-8038$

FUNDACIÓN KOINONIA (F.K)

Santa Ana de Coro, Venezuela.

Maholy Denisse Muñoz Macías; Nicolle Valeria Muñoz Macías; Juan Salvador Solorzano Intriago

\section{TRASPLANTE}

Incompatibilidad no HLA y supervivencia a largo plazo del aloinjerto renal (abril de 2019)

Si bien el desajuste del antígeno leucocitario humano (HLA) de donantes y receptores se ha asociado con peores resultados después del trasplante de riñón, la contribución de la incompatibilidad genética no HLA a la supervivencia del injerto a largo plazo sigue sin estar clara. En un análisis de todo el genoma de 477 pares de donantes fallecidos y primeros receptores de trasplante de riñón con función de injerto estable a los tres meses después del trasplante, se cuantificaron los desajustes genéticos en polimorfismos de nucleótido único no sinónimo (nsSNP) para genes que codifican proteínas transmembrana o secretadas (Reindl et al., 2019). El número de desajustes nsSNP fue predictivo de la pérdida del injerto después de ajustar por desajustes de eplet HLA. Se identificaron anticuerpos específicos de donantes dirigidos contra epítopos desajustados genéticamente para algunas de las proteínas transmembrana entre una cohorte independiente de pares de donantes-receptores con rechazo mediado por anticuerpos crónicos. Aunque se necesitan estudios adicionales para caracterizar aún más estos antígenos no HLA, estos hallazgos respaldan el importante papel de la aloinmunidad no HLA en el trasplante de riñón y su impacto en los resultados del trasplante a largo plazo.

\section{CIRUGÍA DE TRAUMA Y QUEMADURAS}

Colocación del filtro de la vena cava inferior en pacientes con traumatismos gravemente lesionados (julio de 2019)

El uso de filtros profilácticos para la vena cava inferior $(\mathrm{VCl})$ en pacientes con traumatismos graves que presentan una contraindicación para la anticoagulación profiláctica sigue siendo controvertido. En un ensayo aleatorizado que comparó la colocación temprana del filtro de vena cava (dentro de las 72 horas posteriores al ingreso) versus ningún filtro en 240 pacientes, el criterio de valoración combinado de 
Revista Arbitrada Interdisciplinaria de Ciencias de la Salud. SALUD Y VIDA

Volumen 3. Número 6. Año 3. Julio - Diciembre 2019

Hecho el depósito de Ley: FA2016000010

ISSN: $2610-8038$

FUNDACIÓN KOINONIA (F.K)

Santa Ana de Coro, Venezuela.

Maholy Denisse Muñoz Macías; Nicolle Valeria Muñoz Macías; Juan Salvador Solorzano Intriago

embolia pulmonar sintomática (EP) o muerte a los 90 días fue similar en ambos grupos (Ho et al., 2019). Los pacientes que no pudieron ser anticoagulados dentro de los siete días posteriores a la lesión tenían una frecuencia menor de EP si se había colocado un filtro ( 0 versus 15 por ciento sin filtro). Estos datos respaldan nuestra recomendación de no colocar rutinariamente filtros profilácticos de $\mathrm{VCl}$ en pacientes con traumatismos con lesiones graves, sino considerar la colocación de aquellos con un riesgo muy alto con contraindicaciones continuas a la anticoagulación.

\section{CIRUGÍA GASTROINTESTINAL SUPERIOR}

Hernia de hiato después de una esofagectomía abierta y mínimamente invasiva (agosto de 2019)

Las hernias hiatales son una complicación potencialmente molesta de la esofagectomía. En una gran serie retrospectiva que involucró a más de 6000 pacientes sometidos a reparación de una hernia hiatal después de la esofagectomía, la incidencia de hernia hiatal después de una esofagectomía abierta y mínimamente invasiva (MIE) fue de 0,73 y 1,4 por ciento, respectivamente (Gust et al., 2019). La mayor incidencia después de MIE puede deberse a una mayor dificultad para suturar el hiato, especialmente a Ivor Lewis MIE, y a una menor formación de adherencia, lo que permite un mayor movimiento y una posible hernia del contenido abdominal. La reparación laparoscópica de la hernia hiatal postesofagectomía es factible por cirujanos experimentados en circunstancias no urgentes, pero se necesitan más estudios.

\section{Miotomía endoscópica peroral (MEPO) versus dilatación neumática para la acalasia (agosto de 2019)}

La dilatación neumática es la terapia endoscópica primaria para la acalasia. En el primer ensayo aleatorizado que comparó miotomía endoscópica peroral (MEPO) con dilatación neumática en 133 pacientes con acalasia recientemente diagnosticada, MEPO tuvo más éxito a los dos años de seguimiento (puntaje de Eckardt $\leq 3$ y ausencia 
Revista Arbitrada Interdisciplinaria de Ciencias de la Salud. SALUD Y VIDA

Volumen 3. Número 6. Año 3. Julio - Diciembre 2019

Hecho el depósito de Ley: FA2016000010

ISSN: $2610-8038$

FUNDACIÓN KOINONIA (F.K)

Santa Ana de Coro, Venezuela.

Maholy Denisse Muñoz Macías; Nicolle Valeria Muñoz Macías; Juan Salvador Solorzano Intriago

de complicaciones graves o retratamiento: 92 versus 54 por ciento), pero la incidencia de esofagitis por reflujo fue mayor (41 versus 7 por ciento) (Ponds et al., 2019). Un paciente sometido a dilatación neumática tuvo una perforación, que fue el único evento adverso relacionado con el procedimiento en el ensayo. Si estos resultados son validados por otros ensayos, MEPO podría reemplazar la dilatación neumática como la terapia endoscópica primaria para la acalasia.

\section{Gastrectomía distal laparoscópica versus abierta para el cáncer gástrico localmente avanzado (junio de 2019)}

La eficacia de la gastrectomía distal laparoscópica versus abierta para el tratamiento del cáncer gástrico localmente avanzado no está clara. En un ensayo aleatorizado que comparó estos procedimientos en más de 1000 pacientes en China con cáncer gástrico localmente avanzado clínicamente, los resultados (supervivencia general, supervivencia libre de enfermedad, recurrencia acumulativa) fueron similares para ambos enfoques a los tres años de seguimiento (Yu et al., 2019). Sin embargo, estos resultados pueden tener una aplicabilidad limitada a una población occidental debido a que aproximadamente un tercio de los pacientes fueron exagerados (es decir, tenían tumores en estadio I en la patología), ninguno recibió terapia perioperatoria (lo cual es típico en la práctica occidental), y la mayoría de los pacientes occidentales tienen cánceres gástricos proximales (no distales).

Tratamiento de miotomía endoscópica peroral (MEPO) para divertículos de Zenker (mayo de 2019)

Los divertículos de Zenker (ZD) se tratan mediante septotomía quirúrgica o endoscópica. Sin embargo, la septotomía incompleta debido a la incapacidad para delinear el extremo terminal de la ZD puede conducir a recurrencias clínicas en algunos pacientes. La miotomía endoscópica peroral para ZD (Z-MEPO) es una nueva técnica que desarrolla un túnel submucoso para la exposición completa y la disección del 
Revista Arbitrada Interdisciplinaria de Ciencias de la Salud. SALUD Y VIDA

Volumen 3. Número 6. Año 3. Julio - Diciembre 2019

Hecho el depósito de Ley: FA2016000010

ISSN: $2610-8038$

FUNDACIÓN KOINONIA (F.K)

Santa Ana de Coro, Venezuela.

Maholy Denisse Muñoz Macías; Nicolle Valeria Muñoz Macías; Juan Salvador Solorzano Intriago

tabique. En un estudio internacional de 75 pacientes, Z-MEPO tuvo una tasa de éxito técnico general del 97 por ciento, una tasa de eventos adversos del 7 por ciento y una tasa de éxito clínico del 92 por ciento (resolución completa o casi completa de la disfagia sin la necesidad de repetir el procedimiento ) (Yang et al., 2019). Solo un paciente informó recurrencia de los síntomas al año. Cuando la experiencia y el equipo necesarios están disponibles, MEPO es un prometedor tratamiento endoscópico alternativo de ZD que podría facilitar la septotomía completa.

\section{CIRUGÍA VASCULAR Y ENDOVASCULAR}

Nueva directriz global sobre el manejo de la isquemia crónica que amenaza las extremidades (junio de 2019)

La iniciativa Global Vascular Guidelines, que representa los esfuerzos combinados de la Sociedad Europea de Cirugía Vascular, la Sociedad de Cirugía Vascular y la Federación Mundial de Vasculares, ha publicado una nueva guía integral para el tratamiento de la isquemia crónica que amenaza las extremidades (ICAE). Sociedades (Conte et al., 2019). EI ICAE se define como un síndrome clínico de enfermedad arterial periférica con dolor en reposo, gangrena o ulceración de miembros inferiores $>2$ semanas de duración. Este término refleja un espectro de gravedad de la enfermedad, en contraste con el término anterior "isquemia crítica de las extremidades". La guía recomienda usar la clasificación de Herida, Infección, Isquemia del pie (WIfl) para estadificar clínicamente la extremidad e introduce un nuevo sistema de clasificación, el Sistema de estadificación anatómica global de extremidades (GLASS) para estratificar la gravedad anatómica y proporcionar un marco para la extremidad inferior basada en la evidencia. Revascularización de ICAE. También se proporcionan recomendaciones para la intervención endovascular o el bypass quirúrgico de las extremidades inferiores. 
Revista Arbitrada Interdisciplinaria de Ciencias de la Salud. SALUD Y VIDA

Volumen 3. Número 6. Año 3. Julio - Diciembre 2019

Hecho el depósito de Ley: FA2016000010

ISSN: $2610-8038$

FUNDACIÓN KOINONIA (F.K)

Santa Ana de Coro, Venezuela.

Maholy Denisse Muñoz Macías; Nicolle Valeria Muñoz Macías; Juan Salvador Solorzano Intriago

Resultados a largo plazo de la reparación de aneurisma aórtico abdominal abierto versus endovascular (junio de 2019)

Se dispone de datos limitados para los resultados a largo plazo de la reparación endovascular del aneurisma aórtico abdominal (AAA). El ensayo Open Versus Endovascular Repair (OVER) es un ensayo multicéntrico en curso que asignó aleatoriamente a casi 900 veteranos estadounidenses a la reparación endovascular o abierta para AAA> $5.0 \mathrm{~cm}$ (Lederle et al., 2019). Aunque los resultados iniciales parecían favorecer la reparación endovascular, la mortalidad general y la muerte relacionada con el aneurisma fueron similares en ambos grupos seguidos durante hasta 14 años, y significativamente más pacientes endovasculares requirieron procedimientos secundarios. Estos hallazgos respaldan un enfoque individualizado para el tratamiento de AAA.

Preocupaciones sobre la seguridad de los dispositivos recubiertos con paclitaxel para el tratamiento de la enfermedad arterial periférica (abril de 2019)

Los dispositivos recubiertos con paclitaxel se han utilizado con éxito para tratar la enfermedad arterial periférica de las extremidades inferiores (EAP), pero los datos recientes sugieren problemas de seguridad a largo plazo. En la revisión de la Administración de Drogas y Alimentos de los Estados Unidos (ADA) de datos de seguimiento a largo plazo de ensayos aleatorios previos a la comercialización de productos recubiertos con paclitaxel para la EAP, la mortalidad general aumentó a los cinco años después de la colocación de estos dispositivos en comparación con los dispositivos no recubiertos (Administración de Drogas y Alimentos de los Estados Unidos, ADA 2019a). Dadas las limitaciones de los datos disponibles, en lugar de desaconsejar el uso de estos dispositivos, la ADA ha instado a los proveedores de atención médica a considerar los beneficios potenciales junto con los riesgos potenciales para cada individuo antes de usar dispositivos recubiertos con paclitaxel para el tratamiento de la EAP. Estamos de acuerdo con la recomendación de la ADA. 
Revista Arbitrada Interdisciplinaria de Ciencias de la Salud. SALUD Y VIDA

Volumen 3. Número 6. Año 3. Julio - Diciembre 2019

Hecho el depósito de Ley: FA2016000010

ISSN: $2610-8038$

FUNDACIÓN KOINONIA (F.K)

Santa Ana de Coro, Venezuela.

Maholy Denisse Muñoz Macías; Nicolle Valeria Muñoz Macías; Juan Salvador Solorzano Intriago

Apósitos de presión negativa para incisiones en la ingle cerrada después de una cirugía arterial (marzo de 2019)

En comparación con los apósitos convencionales, la terapia de heridas con presión negativa (NPWT) se asocia con tasas reducidas de infección del sitio quirúrgico cuando se usa en incisiones abdominales suturadas. Un metanálisis que incluyó siete ensayos aleatorios y más de 1000 incisiones encontró un beneficio similar después de la cirugía arterial a través de una incisión en la ingle (Svensson et al., 2019). Se necesitan ensayos adicionales para evaluar otros resultados, en particular las complicaciones del injerto, y para determinar la relación costo-efectividad en este entorno clínico.

\section{OTRAS ESPECIALIDADES QUIRÚRGICAS}

Disección de ganglios linfáticos pélvicos extendida versus estándar en cistectomía radical (agosto de 2019)

En estudios observacionales de pacientes con cáncer de vejiga sometidos a cistectomía radical, la disección de ganglios linfáticos pélvicos (DGLP) se ha asociado con mejores resultados oncológicos en comparación con DGLP estándar (linfadenectomía pélvica bilateral). En el primer ensayo aleatorizado que comparó los dos enfoques en más de 400 pacientes con carcinoma urotelial cT1-4aNOM0, el DGLP extendido resultó en tendencias no significativas hacia una mayor supervivencia libre de recurrencia, supervivencia específica del cáncer y supervivencia general a los cinco años (Gschwend et al., 2019). Las limitaciones incluyeron el bajo poder estadístico y la exclusión de pacientes que recibieron quimioterapia neoadyuvante. Los datos de seguimiento más largos y los resultados de un segundo ensayo en curso ayudarán a informar la toma de decisiones sobre la extensión óptima de DGLP en la cistectomía radical. 
Revista Arbitrada Interdisciplinaria de Ciencias de la Salud. SALUD Y VIDA

Volumen 3. Número 6. Año 3. Julio - Diciembre 2019

Hecho el depósito de Ley: FA2016000010

ISSN: $2610-8038$

FUNDACIÓN KOINONIA (F.K)

Santa Ana de Coro, Venezuela.

Maholy Denisse Muñoz Macías; Nicolle Valeria Muñoz Macías; Juan Salvador Solorzano Intriago

Limpieza de meatal con clorhexidina antes de la inserción del catéter uretral (julio de 2019)

El beneficio de la limpieza antiséptica del meato antes de la inserción del catéter uretral es controvertido. En un ensayo aleatorizado de más de 1600 pacientes en hospitales australianos, la limpieza del meato con solución de clorhexidina al 0,1 por ciento versus solución salina normal disminuyó la incidencia de bacteriuria asintomática asociada a la sonda en un 74 por ciento y la infección del tracto urinario en un 94 por ciento (Fasugba et al., 2019). Recomendamos la limpieza antiséptica del meato antes de la inserción del catéter uretral y preferimos usar cualquier agente antiséptico que ya se haya incluido en el kit.

Riesgo infeccioso asociado con productos biológicos y glucocorticoides en el contexto de la artroplastia (junio de 2019)

El riesgo infeccioso asociado con el uso de medicamentos antirreumáticos modificadores de la enfermedad biológica (FARME) en el contexto de la cirugía se evaluó en un estudio utilizando datos de reclamos de seguros de casi 10,000 adultos con artritis reumatoide (AR) sometidos a artroplastia electiva total de rodilla o cadera (George et al., 2019) El riesgo de infección hospitalizada dentro de los 30 días y la infección protésica de las articulaciones dentro de un año fueron similares en todos los productos biológicos. El uso de glucocorticoides se asoció con un aumento dependiente de la dosis tanto en la infección hospitalizada como en la infección de la prótesis articular, particularmente a dosis superiores a $10 \mathrm{mg}$ de prednisona al día. Esto es consistente con nuestro enfoque sugerido de minimizar las dosis de glucocorticoides antes de la cirugía electiva a idealmente menos o igual a $10 \mathrm{mg}$ de prednisona (o equivalente) al día. 
Revista Arbitrada Interdisciplinaria de Ciencias de la Salud. SALUD Y VIDA

Volumen 3. Número 6. Año 3. Julio - Diciembre 2019

Hecho el depósito de Ley: FA2016000010

ISSN: $2610-8038$

FUNDACIÓN KOINONIA (F.K)

Santa Ana de Coro, Venezuela.

Maholy Denisse Muñoz Macías; Nicolle Valeria Muñoz Macías; Juan Salvador Solorzano Intriago

\section{Vasectomía y riesgo de cáncer de próstata (junio de 2019)}

Es controvertido si una vasectomía previa aumenta el riesgo de cáncer de próstata. En un estudio basado en la población de más de dos millones de hombres daneses nacidos entre 1937 y 1966, la vasectomía previa se asoció con un riesgo 15 por ciento mayor de cáncer de próstata que persistió durante al menos 30 años después del procedimiento (Husby, Wohlfahrt y Melbye, 2019). Sin embargo, los estudios de observación no prueban una relación causal entre la vasectomía y el cáncer de próstata, ya que los factores de confusión no pueden excluirse en la selección de hombres que se sometieron al procedimiento. Algunos médicos que realizan vasectomía optan por discutir el pequeño riesgo potencial de cáncer de próstata en aras de una divulgación completa; sin embargo, seguimos las pautas de 2015 de la Asociación Americana de Urología, que establecen que los médicos no necesitan discutir rutinariamente el cáncer de próstata en el asesoramiento previo a la vasectomía.

\section{Falta de beneficio con la radiación después de los procedimientos de la pared torácica para el mesotelioma pleural maligno (mayo de 2019)}

En el mesotelioma pleural maligno, se ha sugerido la radioterapia profiláctica (RP) al sitio del tracto después de procedimientos limitados de la pared torácica ( $\mathrm{p}$. Ej., Biopsias quirúrgicas abiertas, toracoscopia, drenajes torácicos, etc.) como una forma de prevenir la siembra tumoral, pero esta estrategia es controvertida. En un ensayo aleatorizado que evaluó la RT profiláctica posterior al procedimiento en 375 pacientes con mesotelioma pleural maligno que se habían sometido al procedimiento de la pared torácica en las últimas seis semanas, no hubo diferencias en las tasas de metástasis en la pared torácica a los seis meses entre la RT y ninguna RT grupos (Bayman et al., 2019). Teniendo en cuenta estos y los resultados anteriores, sugerimos no usar de manera rutinaria radiación profiláctica en el sitio del tracto después de los procedimientos de la pared torácica para el mesotelioma pleural maligno. 


\section{Malla prohibida para la reparación del prolapso de órganos pélvicos transvaginales en EE. UU. (Abril de 2019)}

Las agencias reguladoras de todo el mundo han publicado diversas recomendaciones sobre el uso de malla sintética en el tratamiento del prolapso de órganos pélvicos (POP). En abril de 2019, la Administración de Alimentos y Medicamentos de EE. UU. Prohibió la venta y distribución de mallas quirúrgicas sintéticas para la reparación transvaginal de POP porque no pudieron confirmar que los beneficios probables superaran los riesgos probables (Administración de Drogas y Alimentos de los Estados Unidos, 2019c). Esta prohibición no aplica a la reparación transabdominal de POP ni al tratamiento de la incontinencia urinaria de esfuerzo (IUE). No se necesita ninguna acción en mujeres a las que se les ha insertado una malla transvaginal para reparar el POP y están satisfechas con el resultado. Aquellos que tienen complicaciones o síntomas deben notificar a su proveedor de atención médica.

\section{REFERENCIAS BIBLIOGRÁFICAS}

1. Administración de Drogas y Alimentos de los Estados Unidos (2019a). Tratamiento de la enfermedad arterial periférica con globos recubiertos con paclitaxel y stents liberadores de paclitaxel potencialmente asociados con una mayor mortalidad. https://www.fda.gov/medical-devices/letters-health-careproviders/august-7-2019-update- tratamiento-enfermedad-periférica-arterialpaclitaxel-cubierto-globos-y-paclitaxel (Consultado el 04 de septiembre de 2019).

2. Administración de Drogas y Alimentos de los Estados Unidos. (2019b). La FDA toma medidas para proteger a las pacientes del riesgo de ciertos implantes mamarios con textura; solicita que Allergan retire voluntariamente ciertos implantes mamarios y expansores de tejidos del mercado. https://www.fda.gov/news-events/press-announcements/fda-takes-action-protectpatients-risk-certain-textured-breast-implants-requests-allergan (consultado el 24 de agosto de 2019).

3. Administración de Drogas y Alimentos de los Estados Unidos. (2019c). Abril de 2019. Implantes

de malla quirúrgica uroginecológica. 
www.fda.gov/MedicalDevices/ProductsandMedicalProcedures/ImplantsandProst hetics/UroGynSurgicalMesh/default.htm (consultado el 18 de agosto de 2019).

4. Bayman N, Appel W, Ashcroft L, et al. (2019). Irradiación profiláctica de tractos en pacientes con mesotelioma pleural maligno: un ensayo aleatorizado, multicéntrico, de fase III aleatorizado. J Clin Oncol

5. Bednarski BK, Nickerson TP, You YN, et al. (2019). Ensayo clínico aleatorizado de recuperación acelerada mejorada después de una cirugía de cáncer colorrectal mínimamente invasiva (ensayo RecoverMI). Br J Surg

6. Bellin MD, Beilman GJ, Sutherland DE, et al. (2019).¿Qué tan duraderas son la pancreatectomía total y el trasplante de células de los islotes intraportales para el tratamiento de la pancreatitis crónica? J Am Coll Surg

7. Bersoff SJ, Chamberlain C, Cao C, et al. (2019). Gangrena de Fournier asociada con inhibidores del cotransportador-2 de glucosa y sodio: una revisión de los casos espontáneos posteriores a la comercialización. Ann Intern Med

8. Chan MTV, Wang CY, Seet E, et al. (2019). Asociación de apnea obstructiva del sueño no reconocida con eventos cardiovasculares postoperatorios en pacientes sometidos a cirugía mayor no cardíaca. JAMA

9. Chapman WC Jr, Subramanian M, Jayarajan S, et al. (2019). Primero, no hacer daño: repensar el desvío de rutina en la resección del cáncer de recto que preserva el esfínter. J Am Coll Surg

10. Comité de redacción para el grupo colaborativo PROBESE de la red de ventilación PROtective (PROVEnet) para la red de ensayos clínicos de la Sociedad Europea de Anestesiología, Bluth T, Serpa Neto A, et al. (2019). Efecto de la presión intraoperatoria positiva al final de la espiración (PEEP) intraoperatoria con maniobras de reclutamiento versus PEEP baja sobre las complicaciones pulmonares postoperatorias en pacientes obesos: un ensayo clínico aleatorizado. JAMA

11. Conte MS, Bradbury AW, Kolh P, et al. (2019). Pautas vasculares globales sobre el manejo de la isquemia crónica que amenaza las extremidades. J Vasc Surg

12. Dakik HA, Chehab O, Eldirani $M$, et al. (2019). Un nuevo índice para la evaluación cardiovascular preoperatoria. J Am Coll Cardiol 2019; 73: 3067. 
13. Fasugba O, Cheng AC, Gregory V, et al. (2019). Clorhexidina para la limpieza del meato en la reducción de las infecciones del tracto urinario asociadas a la sonda: un ensayo controlado aleatorio de cuña escalonada multicéntrico Lancet Infect Dis

14. Fleshman J, Branda ME, Sargent DJ, et al. (2019). Supervivencia libre de enfermedad y recurrencia local para la resección laparoscópica en comparación con la resección abierta de cáncer rectal en estadios II a III: resultados del seguimiento del ensayo controlado aleatorizado ACOSOG Z6051. Ann Surg

15. George MD, Baker JF, Winthrop K, et al. (2019). Riesgo de productos biológicos y glucocorticoides en pacientes con artritis reumatoide sometidos a artroplastia: un estudio de cohorte. Ann Intern Med.

16. Goldstone RN, Cauley CE, Chang DC, et al. (2019). El efecto del entrenamiento quirúrgico y el enfoque operativo sobre los resultados en la diverticulitis aguda: ¿deberían revisarse las pautas? Dis Colon Rectum

17. Gschwend JE, Heck MM, Lehmann J, et al. (2019). Disección extendida versus limitada de ganglios linfáticos en pacientes con cáncer de vejiga sometidos a cistectomía radical: resultados de supervivencia de un ensayo prospectivo aleatorizado. Eur Urol

18. Guldvog I, Reitsma LC, Johnsen L, et al. (2019). Tiroidectomía versus tratamiento médico para pacientes eutiroideos con enfermedad de Hashimoto y síntomas persistentes: un ensayo aleatorizado. Ann Intern Med.

19. Gust L, Nafteux P, Allemann P, et al. (2019). Hernia de hiato después de la esofagectomía: una gran encuesta europea. Eur J Cardiothorac Surg

20. Gutlic N, Gutlic A, Petersson U, et al. (2019). Ensayo clínico aleatorizado que compara la reparación extraperitoneal total con la reparación de hernia inguinal de Lichtenstein (ensayo TEPLICH). Br J Surg.

21. Ho KM, Rao S, Honeybul S, et al. (2019). Un ensayo multicéntrico de filtros de vena cava en pacientes gravemente heridos. $\mathrm{N}$ Engl J Med

22. https://www.auanet.org/guidelines/vasectomy-(2012-amended-2015) (consultado el 13 de junio de 2017).

23. Huang SS, Septimus E, Kleinman K, et al. (2019). Clorhexidina versus baños de rutina para prevenir organismos resistentes a múltiples medicamentos e 
Revista Arbitrada Interdisciplinaria de Ciencias de la Salud. SALUD Y VIDA

Volumen 3. Número 6. Año 3. Julio - Diciembre 2019

Hecho el depósito de Ley: FA2016000010

ISSN: $2610-8038$

FUNDACIÓN KOINONIA (F.K).

Santa Ana de Coro, Venezuela.

Maholy Denisse Muñoz Macías; Nicolle Valeria Muñoz Macías; Juan Salvador Solorzano Intriago

infecciones del torrente sanguíneo por todas las causas en unidades médicas y quirúrgicas generales (ensayo de infección ABATE): un ensayo aleatorizado por conglomerados. Lancet

24. Husby A, Wohlfahrt J, y Melbye M. (2019). Vasectomía y riesgo de cáncer de próstata: un estudio de cohorte nacional de 38 años. J Natl Cancer Inst.

25. Inge TH, Courcoulas AP, Jenkins TM, et al. (2019). Resultados a cinco años del bypass gástrico en adolescentes en comparación con los adultos. $\mathrm{N}$ Engl J Med.

26. Karakatsanis A, Hersi AF, Pistiolis L, et al. (2019). Efecto de la inyección preoperatoria de partículas de óxido de hierro superparamagnético sobre las tasas de disección de ganglios linfáticos centinela en mujeres sometidas a cirugía por carcinoma ductal in situ (estudio SentiNot). Br J Surg

27. Keane C, Park J, Öberg S, et al. (2019). Resultados funcionales de un ensayo aleatorizado de cierre temprano de ileostomía temporal después de la escisión rectal para el cáncer. Br J Surg

28. Kohler A, Lavanchy JL, Lenoir $U$, et al. (2019). Eficacia de la implantación profiláctica de malla intraperitoneal para la prevención de la hernia incisional en pacientes sometidos a cirugía abdominal abierta: un ensayo clínico aleatorizado. JAMA Surg

29. Kondo N, Uemura K, Nakagawa N, et al. (2019). Un ensayo multicéntrico, aleatorizado y controlado que compara las engrapadoras reforzadas con las engrapadoras desnudas durante la pancreatectomía distal (ensayo HiSCO-07). Ann Surg Oncol

30. Lederle FA, Kyriakides TC, Stroupe KT, et al. (2019). Reparación abierta versus endovascular del aneurisma aórtico abdominal. N Engl J Med

31. Lerman BJ, Popat RA, Assimes TL, et al. (2019). Asociación de la fracción de eyección del ventrículo izquierdo y los síntomas con la mortalidad después de la cirugía electiva no cardíaca entre pacientes con insuficiencia cardíaca. JAMA

32. Mällinen J, Rautio T, Grönroos J, et al. (2019). Riesgo de neoplasia apendicular en el absceso periappendicular en pacientes tratados con apendicectomía de intervalo versus seguimiento con imágenes de resonancia magnética: resultados de 1 año del ensayo clínico aleatorizado de periapendicitis aguda. JAMA Surg 
33. Mari GM, Crippa J, Cocozza E, et al. (2019). La ligadura baja de la arteria mesentérica inferior en la resección anterior laparoscópica por cáncer rectal reduce la disfunción genitourinaria: resultados de un ensayo controlado aleatorio (ensayo HIGHLOW). Ann Surg

34. Mocanu V, Dang JT, Ladak F, et al. (2019). Uso de antibióticos en la prevención de fístulas anales después de la incisión y el drenaje de abscesos anorrectales: una revisión sistemática y un metanálisis. Am J Surg.

35. Nordholm A, Schnack M, y Krarup PM. (2019). Aumento de las tasas de fuga después de la anastomosis ileocólica grapada versus cosida a mano en pacientes con cáncer de colon del lado derecho: un estudio de cohorte a nivel nacional. Dis Colon Rectum

36. Odensten C, Strigård K, Rutegård J, et al. (2019). El uso de malla profiláctica al crear una colostomía no previene la hernia paraestomal: un ensayo controlado aleatorio-STOMAMESH. Ann Surg

37. Ponds FA, Fockens $P$, Lei $A$, et al. (2019). Efecto de la miotomía endoscópica peroral versus dilatación neumática sobre la gravedad de los síntomas y los resultados del tratamiento entre pacientes sin tratamiento previo con acalasia: un ensayo clínico aleatorizado. JAMA

38. Reindl R, Heinzel A, Kainz A, et al. (2019). Contribución de la incompatibilidad no HLA entre el donante y el receptor a la supervivencia del aloinjerto renal: análisis de todo el genoma en una cohorte prospectiva. Lancet

39. Robert M, Espalieu P, Pelascini E, et al. (2019).Eficacia y seguridad de un bypass gástrico de anastomosis versus bypass gástrico Roux-en- $Y$ para obesidad (YOMEGA): un ensayo multicéntrico, aleatorizado, abierto, de no inferioridad. Lancet

40. Rottier SJ, van Dijk ST, van Geloven AAW, et al. (2019). Metaanálisis del papel de la colonoscopia después de un episodio de diverticulitis aguda del lado izquierdo. Br J Surg

41. Schulman PM, Treggiari MM, Yanez ND, et al. (2019). Interferencia electromagnética con posicionamiento de electrodo dispersivo de electrocirugía protocolo en pacientes con desfibriladores cardioversores implantables. Anestesiología 
42. Stevenson ARL, Solomon MJ, Brown CSB, et al. (2019). Supervivencia libre de enfermedad y recurrencia local después de la resección asistida por laparoscopia o la resección abierta para el cáncer de recto: el ensayo clínico aleatorizado de cáncer laparoscópico del recto de Australasia. Ann Surg

43. Svensson R, Zarrouk M, Asciutto G, et al. (2019). Metanálisis de la terapia de herida por presión negativa de incisiones inguinales cerradas en cirugía arterial. $\mathrm{Br} J$ Surg

44. Utjés D, Malmstedt J, Teras J, et al. (2019). Márgenes de escisión quirúrgica de $2 \mathrm{~cm}$ versus $4 \mathrm{~cm}$ para melanoma cutáneo primario más grueso de $2 \mathrm{~mm}$ : seguimiento a largo plazo de un ensayo aleatorizado multicéntrico. Lancet

45. Van Dijk AH, Wennmacker SZ, de Reuver PR, et al. (2019). Estrategia restrictiva versus atención habitual para la colecistectomía en pacientes con cálculos biliares y dolor abdominal (SECURE): un ensayo multicéntrico, aleatorizado, de brazo paralelo, de no inferioridad. Lancet

46. Van Hilst J, de Rooij T, Bosscha K, et al. (2019). Pancreatoduodenectomía laparoscópica versus abierta para tumores pancreáticos o periampulares (LEOPARD-2): un ensayo multicéntrico, cegado al paciente, aleatorizado, controlado en fase 2/3. Lancet Gastroenterol Hepatol

47. Yang J, Novak S, Ujiki M, et al. (2019). Un estudio internacional sobre el uso de miotomía endoscópica peroral en el tratamiento del divertículo de Zenker. Gastrointest Endosc

48. Yu J, Huang C, Sun Y, et al. (2019). Efecto de la gastrectomía distal laparoscópica versus abierta en la supervivencia sin enfermedad a los 3 años en pacientes con cáncer gástrico localmente avanzado: el ensayo clínico aleatorizado CLASS-01. JAMA 
1. United States Food and Drug Administration (2019a). Treatment of peripheral arterial disease with paclitaxel-coated balloons and paclitaxel-releasing stents potentially associated with increased mortality. https://www.fda.gov/medicaldevices/letters-health-care-providers/august-7-2019-updatetreatmentperipheral-arterial-disease-paclitaxel-covered-balloons-and-paclitaxel (Accessed on September 4, 2019).

2. United States Food and Drug Administration. (2019b). The FDA takes measures to protect patients from the risk of certain textured breast implants; Allergan asks to voluntarily remove certain breast implants and tissue expanders from the market. https://www.fda.gov/news-events/press-announcements/fda-takesaction-protect-patients-risk-certain-textured-breast-implants-requests-allergan (accessed August 24, 2019).

3. United States Food and Drug Administration. (2019c). April 2019. Urogynecological surgical mesh implants. www.fda.gov/MedicalDevices/ProductsandMedicalProcedures/ImplantsandProst hetics/UroGynSurgicalMesh/default.htm (accessed August 18, 2019).

4. Bayman N, Appel W, Ashcroft L, et al. (2019). Prophylactic irradiation of tracts in patients with malignant pleural mesothelioma: a randomized, multicenter, randomized phase III trial. J Clin Oncol

5. Bednarski BK, Nickerson TP, You YN, et al. (2019). Randomized clinical trial of improved accelerated recovery after minimally invasive colorectal cancer surgery (RecoverMI trial). Br J Surg

6. Bellin MD, Beilman GJ, Sutherland DE, et al. (2019). How durable are total pancreatectomy and intraportal islet cell transplantation for the treatment of chronic pancreatitis? J Am Coll Surg

7. Bersoff SJ, Chamberlain C, Cao C, et al. (2019). Fournier gangrene associated with glucose and sodium cotransporter-2 inhibitors: a review of post-marketing spontaneous cases. Ann Intern Med

8. Chan MTV, Wang CY, Seet E, et al. (2019). Association of obstructive sleep apnea not recognized with postoperative cardiovascular events in patients undergoing major non-cardiac surgery. JAMA 
9. Chapman WC Jr, Subramanian M, Jayarajan S, et al. (2019). First, do no harm: rethink the routine deviation in the resection of rectal cancer that preserves the sphincter. J Am Coll Surg

10. Drafting committee for the PROBESE collaborative group of the PROtective ventilation network (PROVEnet) for the clinical trials network of the European Society of Anesthesiology, Bluth T, Serpa Neto A, et al. (2019). Effect of positive intraoperative pressure at the end of expiration (PEEP) intraoperatively with recruitment maneuvers versus low PEEP on postoperative pulmonary complications in obese patients: a randomized clinical trial. JAMA

11. Conte MS, Bradbury AW, Kolh $P$, et al. (2019). Global vascular guidelines on the management of chronic ischemia that threatens the extremities. J Vasc Surg

12. Dakik HA, Chehab O, Eldirani M, et al. (2019). A new index for preoperative cardiovascular evaluation. J Am Coll Cardiol 2019; 73: 3067.

13. Fasugba O, Cheng AC, Gregory V, et al. (2019). Chlorhexidine for cleansing the meatus in reducing urinary tract infections associated with the probe: a randomized controlled trial of multicenter staggered wedge Lancet Infect Dis

14. Fleshman J, Branda ME, Sargent DJ, et al. (2019). Disease-free survival and local recurrence for laparoscopic resection compared to open resection of stage II to III rectal cancer: results of the follow-up of the ACOSOG Z6051 randomized controlled trial. Ann Surg

15. George MD, Baker JF, Winthrop K, et al. (2019). Risk of biological and glucocorticoid products in patients with rheumatoid arthritis undergoing arthroplasty: a cohort study. Ann Intern Med.

16. Goldstone RN, Cauley CE, Chang DC, et al. (2019). The effect of surgical training and the operational approach on outcomes in acute diverticulitis: should the guidelines be reviewed? Dis Colon Rectum

17. Gschwend JE, Heck MM, Lehmann J, et al. (2019). Extended versus limited lymph node dissection in patients with bladder cancer undergoing radical cystectomy: survival results of a prospective randomized trial. Eur Urol

18. Guldvog I, Reitsma LC, Johnsen L, et al. (2019). Thyroidectomy versus medical treatment for euthyroid patients with Hashimoto's disease and persistent symptoms: a randomized trial. Ann Intern Med. 
19. Gust L, Nafteux P, Allemann P, et al. (2019). Hiatus hernia after esophagectomy: a large European survey. Eur J Cardiothorac Surg

20. Gutlic N, Gutlic A, Petersson U, et al. (2019). Randomized clinical trial comparing total extraperitoneal repair with Lichtenstein inguinal hernia repair (TEPLICH trial). Br J Surg.

21. Ho KM, Rao S, Honeybul S, et al. (2019). A multicenter trial of vena cava filters in seriously injured patients. N Engl J Med

22. https://www.auanet.org/guidelines/vasectomy-(2012-amended-2015) (accessed June 13, 2017).

23. Huang SS, Septimus E, Kleinman K, et al. (2019). Chlorhexidine versus routine baths to prevent multi-drug resistant organisms and bloodstream infections from all causes in general medical and surgical units (ABATE infection test): a cluster randomized trial. Lancet

24. Husby A, Wohlfahrt J, and Melbye M. (2019). Vasectomy and risk of prostate cancer: a 38-year national cohort study. J Natl Cancer Inst.

25. Inge TH, Courcoulas AP, Jenkins TM, et al. (2019). Five-year results of gastric bypass in adolescents compared to adults. $\mathrm{N}$ Engl $\mathrm{J}$ Med.

26. Karakatsanis A, Hersi AF, Pistiolis L, et al. (2019). Effect of preoperative injection of superparamagnetic iron oxide particles on sentinel lymph node dissection rates in women undergoing surgery for ductal carcinoma in situ (SentiNot study). $\mathrm{Br} J$ Surg

27. Keane C, Park J, Öberg S, et al. (2019). Functional results of a randomized trial of early closure of temporary ileostomy after rectal excision for cancer. $\mathrm{Br} \mathrm{J}$ Surg

28. Kohler A, Lavanchy JL, Lenoir U, et al. (2019). Efficacy of prophylactic intraperitoneal mesh implantation for the prevention of incisional hernia in patients undergoing open abdominal surgery: a randomized clinical trial. JAMA Surg

29. Kondo N, Uemura K, Nakagawa N, et al. (2019). A multicenter, randomized and controlled trial comparing reinforced staplers with bare staplers during distal pancreatectomy (HiSCO-07 trial). Ann Surg Oncol 
Revista Arbitrada Interdisciplinaria de Ciencias de la Salud. SALUD Y VIDA

Volumen 3. Número 6. Año 3. Julio - Diciembre 2019

Hecho el depósito de Ley: FA2016000010

ISSN: $2610-8038$

FUNDACIÓN KOINONIA (F.K).

Santa Ana de Coro, Venezuela.

Maholy Denisse Muñoz Macías; Nicolle Valeria Muñoz Macías; Juan Salvador Solorzano Intriago

30. Lederle FA, Kyriakides TC, Stroupe KT, et al. (2019). Open versus endovascular repair of abdominal aortic aneurysm. $\mathrm{N}$ Engl J Med

31. Lerman BJ, Popat RA, Assimes TL, et al. (2019). Association of left ventricular ejection fraction and symptoms with mortality after elective non-cardiac surgery among patients with heart failure. JAMA

32. Mällinen J, Rautio T, Grönroos J, et al. (2019). Risk of appendicular neoplasia in periappendicular abscess in patients treated with interval appendectomy versus follow-up with magnetic resonance imaging: 1 year results of the randomized clinical trial of acute periapendicitis. JAMA Surg

33. Mari GM, Crippa J, Cocozza E, et al. (2019). Low ligation of the inferior mesenteric artery in laparoscopic anterior resection for rectal cancer reduces genitourinary dysfunction: results of a randomized controlled trial (HIGHLOW trial). Ann Surg

34. Mocanu V, Dang JT, Ladak F, et al. (2019). Antibiotic use in the prevention of anal fistulas after incision and drainage of anorectal abscesses: a systematic review and a meta-analysis. Am J Surg.

35. Nordholm A, Schnack M, and Krarup PM. (2019). Increased leakage rates after stapled versus hand-sewn ileocolic anastomosis in patients with right-sided colon cancer: a nationwide cohort study. Dis Colon Rectum.

36. Odensten C, Strigård K, Rutegård J, et al. (2019). El uso de malla profiláctica al crear una colostomía no previene la hernia paraestomal: un ensayo controlado aleatorio-STOMAMESH. Ann Surg

37. Ponds FA, Fockens $P$, Lei $A$, et al. (2019). Efecto de la miotomía endoscópica peroral versus dilatación neumática sobre la gravedad de los síntomas y los resultados del tratamiento entre pacientes sin tratamiento previo con acalasia: un ensayo clínico aleatorizado. JAMA

38. Reindl R, Heinzel A, Kainz A, et al. (2019). Contribución de la incompatibilidad no HLA entre el donante y el receptor a la supervivencia del aloinjerto renal: análisis de todo el genoma en una cohorte prospectiva. Lancet

39. Robert M, Espalieu P, Pelascini E, et al. (2019).Eficacia y seguridad de un bypass gástrico de anastomosis versus bypass gástrico Roux-en-Y para obesidad (YOMEGA): un ensayo multicéntrico, aleatorizado, abierto, de no inferioridad. Lancet 
40. Rottier SJ, van Dijk ST, van Geloven AAW, et al. (2019). Metaanálisis del papel de la colonoscopia después de un episodio de diverticulitis aguda del lado izquierdo. Br J Surg

41. Schulman PM, Treggiari MM, Yanez ND, et al. (2019). Interferencia electromagnética con posicionamiento de electrodo dispersivo de electrocirugía protocolo en pacientes con desfibriladores cardioversores implantables. Anestesiología

42. Stevenson ARL, Solomon MJ, Brown CSB, et al. (2019). Supervivencia libre de enfermedad y recurrencia local después de la resección asistida por laparoscopia o la resección abierta para el cáncer de recto: el ensayo clínico aleatorizado de cáncer laparoscópico del recto de Australasia. Ann Surg

43. Svensson R, Zarrouk M, Asciutto G, et al. (2019). Metanálisis de la terapia de herida por presión negativa de incisiones inguinales cerradas en cirugía arterial. $\mathrm{Br} J$ Surg

44. Utjés D, Malmstedt J, Teras J, et al. (2019). Márgenes de escisión quirúrgica de $2 \mathrm{~cm}$ versus $4 \mathrm{~cm}$ para melanoma cutáneo primario más grueso de $2 \mathrm{~mm}$ : seguimiento a largo plazo de un ensayo aleatorizado multicéntrico. Lancet

45. Van Dijk AH, Wennmacker SZ, de Reuver PR, et al. (2019). Estrategia restrictiva versus atención habitual para la colecistectomía en pacientes con cálculos biliares y dolor abdominal (SECURE): un ensayo multicéntrico, aleatorizado, de brazo paralelo, de no inferioridad. Lancet

46. Van Hilst J, de Rooij T, Bosscha K, et al. (2019). Pancreatoduodenectomía laparoscópica versus abierta para tumores pancreáticos o periampulares (LEOPARD-2): un ensayo multicéntrico, cegado al paciente, aleatorizado, controlado en fase 2/3. Lancet Gastroenterol Hepatol

47. Yang J, Novak S, Ujiki M, et al. (2019). Un estudio internacional sobre el uso de miotomía endoscópica peroral en el tratamiento del divertículo de Zenker. Gastrointest Endosc

48. Yu J, Huang C, Sun Y, et al. (2019). Efecto de la gastrectomía distal laparoscópica versus abierta en la supervivencia sin enfermedad a los 3 años en pacientes con cáncer gástrico localmente avanzado: el ensayo clínico aleatorizado CLASS-01. JAMA 
Revista Arbitrada Interdisciplinaria de Ciencias de la Salud. SALUD Y VIDA

Volumen 3. Número 6. Año 3. Julio - Diciembre 2019

Hecho el depósito de Ley: FA2016000010

ISSN: $2610-8038$

FUNDACIÓN KOINONIA (F.K)

Santa Ana de Coro, Venezuela.

Maholy Denisse Muñoz Macías; Nicolle Valeria Muñoz Macías; Juan Salvador Solorzano Intriago

@2019 por los autores. Este artículo es de acceso abierto y distribuido según los términos y condiciones de la licencia Creative Commons Atribución-NoComercial-Compartirlgual 4.0 Internacional (CC BY-NC-

SA 4.0) (https://creativecommons.org/licenses/by-nc-sa/4.0/). 